\title{
LADM AND INTERLIS AS A PERFECT MATCH FOR 3D CADASTRE
}

\author{
Eftychia Kalogianni $^{\mathrm{a}, \mathrm{b}}$ *, Efi Dimopoulou ${ }^{\mathrm{b}}$, Wilko Quak $^{\mathrm{a}}$ and Peter Van Oosterom ${ }^{\mathrm{a}}$ \\ ${ }^{a}$ Delft University of Technology, Julianalaan 134, 2628 BL Delft, the Netherlands - (c.w.quak, P.J.M.vanOosterom) @tudelft.nl \\ ${ }^{\mathrm{b}}$ National Technical University of Athens, 9, Iroon Polytechneiou, 15780 Athens, Greece- \\ efkaloyan@gmail.com \& efi@survey.ntua.gr
}

Commission VI, WG VI/4

KEY WORDS: LADM, INTERLIS language, constraints, code lists, legal and physical integration

\begin{abstract}
:
Standardization in land administration domain has been expanded to 3D and even 4D representations, adopting a multipurpose character, in order to become the foundation of a sustainable and smart economic development. At the moment, although the potential benefits of 3D Cadastre is argued to be enormous and there are plenty of standards related to 3D Cadastre while others enhancing the role of 3D Cities, there is no complete solution for 3D Cadastre. That being so, the last years, there has been a rapid increase in the integration, harmonization and implementation support of such standards. In this context, the integration of 3D legal spaces with 3D physical objects is gaining ground, as the (invisible) legal boundaries do not always match with the physical counterparts, leading to obscure situations. LADM, the International Standard for land administration, was proved to be one of the best candidates to unambiguously represent 3D Rights, Restrictions and Responsibilities. On the other side, spatial data models and virtual city models manage 3D urban structures without focusing on legal aspects. Many researchers have explored integrations between those aspects giving promising results. In this direction, apart from international standards, also national standards have been developed to enable the communication between land information systems. One of the most representatives is INTERLIS, a Swiss standard, a precise, standardized Object Relational modelling language on the conceptual level, which allows for automated quality control. Thus, in this paper the focus is given on how INTERLIS and LADM complement each other in the actual implementation of land administration systems. Main challenges among others in the context of this research include: 1. extensible hierarchical and versioned code lists in INTERLIS models, 2. formally define LADM constraints in INTERLIS, 3. discuss 3D geometry types and 4. introduce a holistic LADM/INTERLIS approach for country profiles.
\end{abstract}

\section{INTRODUCTION}

Taking advantage of the developments in 3D geo-information, multiple standards have been developed as domain specific standardization, much needed to capture the semantics of land administration field. Harmonizing and integrating such standards and models in order to support registrations and land administration procedures using Geographic Information Systems along with Data Base Management Systems and applications is crucial.

Some of these standards focus only on the physical, geometrical and functional characteristics of urban structures, while others mostly support management of legal information. Pointing at the maximum usage of such standards has led into the investigation of the integration of legal and physical models through combined approaches.

Current discussions and efforts focus on this integration, where the Land Administration Domain Model (LADM) mainly represents the legal aspect of the models, while CityGML (using ADEs) (Groger et al., 2012), BIM (NBIMS, 2012) and InfraGML (Scarponcini et al., 2016) are usually used to describe the physical counterparts. Over the last years, several projects suggested describing LADM country profiles with the conceptual schema language INTERLIS. This occurs from INTERLIS competitive advantage, as it offers a wide range of tools for automatic control and validation of the data.

Heading towards integrated models, semantically rich elements, as well as constraints play an important role. Hence, this paper, being part of a wider research study (Kalogianni E. et al., 2015, Kalogianni et al., 2016), aims at leveraging INTERLIS as a tool to formally describe constraints and model semantically enriched code lists.

The rest of the paper is organized as follows. In the next section the necessary background information is briefly presented. Following, Section 3 introduces the research work that has been carried out regarding LADM - INTERLIS integration, while the last section presents the conclusions of this research and future trends in this field.

\section{BACKGROUND INFORMATION}

\subsection{Research Background}

Juridical boundaries in 3D are not always bound by physical borders, and only the synergy of 3D visualization together with the clear division of property interests can assure legal certainty. An indicative example where 3D boundaries do not coincide with physical objects and the air space plays an important role is presented in Thompson et al., 2015 (figures 4 and 5). It is therefore evident that the evaluation and validation of 3D legal and physical data, both separately and together at an integrated model is vital.

Despite the recent developments in the field of 3D Cadastres, confusion still exists over terminology and key concepts. For instance, the term 3D SDI refers to the concept of an information infrastructure that includes both 3D legal information and 3D representations of physical real-world objects (Janečka et al. 2016). However, more than one concepts can be generally described with this definition and therefore, meaningful communication should be enabled by using existing standards and by further discussing terminology and concepts. Hence, semantic technologies (ontologies, RDF, etc.) have been proven 
vital for the legal- physical integration and can also be used in land administration to further provide explicit meaning to code list values in more refine manner than just a hierarchy (Lemmen et al., 2014).

2.1.1 LADM implementations: The Land Administration Domain Model (LADM) is the result of different ideas embodied in Cadastre 2014 (Lemmen et al., 2015) and subject to extensive international expert discussions with FIG and ISO TC211 which can be expanded to provide an extendable and adaptable fundament for efficient and effective development, based on Model Driven Architecture (MDA) (Lemmen et al., 2014). Consequently, multiple LADM - based country profiles implementations have been developed since the approval of LADM as an ISO standard in 2012.

Over the last years, more and more standards are being used to implement LADM profiles in the context of projects, some of them being synergies between universities working together with manufacturers in putting research into practice.

In this context, this integration of legal and physical models has been investigated over the last years and various approaches are being developed. A comprehensive review of the approaches towards the integration of legal (usually using LADM as reference) and physical models is presented in (Atazadeh et al., 2016).

2.1.2 INTERLIS concept and tools: INTERLIS is a wellestablished Swiss national standard (SN 612030) for geoinformation exchange, modelling and integration of geo-data allowing cooperation between information systems and especially geographic information systems (KOGIS, 2006). INTERLIS version 2.3 is an object-oriented conceptual schema language, "which allows to precisely describe data models in a textual form and with a rigid computer-processable syntax" (Germann et al., 2014).

What make INTERLIS usage unique, among others, is the formal description of constraints using an OCL - like language and the ability to quality check INTERLIS data against INTERLIS data models using tools enabling automated validation of data. INTERLIS is vendor independent, and for its implementation a tool chain of Java programs is provided to automatically generate implementation components for specific environments, has been developed (Germann et al., 2014).

During data modelling phase INTERLIS UML Editor can be used to generate automatically INTERLIS model files (*.ili files) from UML diagrams. In order to validate the resulted model, INTERLIS offers 3 tools: INTERLIS Compiler (ili2c), which validates the syntactical correctness and semantic compliance of INTERLIS data models, INTERLIS Checker (igchecker2), which is used to quality check INTERLIS XML data against INTERLIS data models and INTERLIS Validator (iliValidator), the open source alternative to INTERLIS Checker (Jenni et al., 2017).

In order to facilitate the translation of the object-oriented INTERLIS models and the relational databases ObjectRelational mappings the following tools have been developed: INTERLIS 2 loader for PostgreSQL/ PostGIS (ili2pg), INTERLIS 2 loader for Oracle (ili2ora) and INTERLIS loader for OGC Geopackage (ili2gpgk). Last but not least, the LADM Project Generator and Editing plugin for QGIS is a tool currently under development, which together with the INTERLIS Reader/
Writer to FME (ilii2fme) comprise the INTERLIS tools for the implementation phase (Jenni et al., 2017).

The INTERLIS tools for each phase are illustrated in Figure 1.

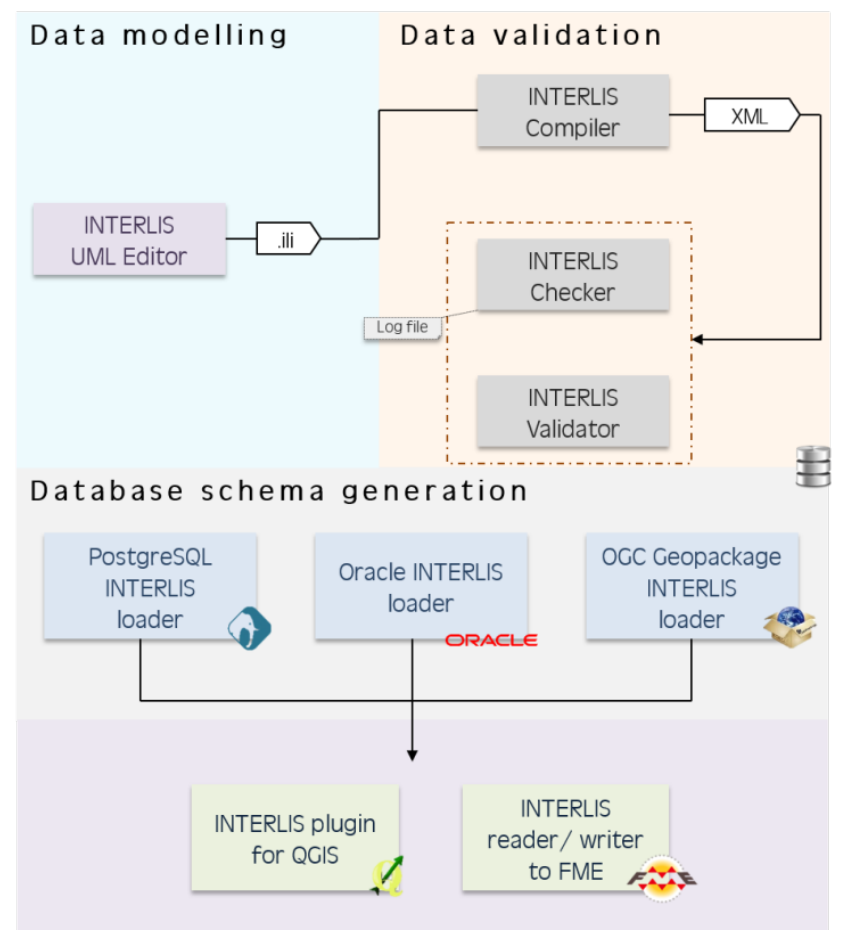

Figure 1. INTERLIS tools

\section{LADM - INTERLIS APPROACH}

Both the international standard LADM and the national standard INTERLIS share the same MDA principles (Germann et al., 2014). Thus, the last years, more and more projects (Germann et al., 2014; Kalogianni et al., 2016; Jenni et al., 2017) suggest the description of LADM country profiles with INTERLIS, exploiting the possibilities of automated control and validation through its tools.

The INTERLIS tools used for the purpose of this research are: INTERLIS Compiler (ili2c), INTERLIS Checker (igchecker2) and INTERLIS loader for PostgreSQL (ili2pg). Hence, the main focus was, as presented below, at the description of code lists, the formal specification of constraints, as well the introduction of a $3 \mathrm{D}$ data type in INTERLIS language.

Initially, Swiss Land Management (SLM) has started an initiative to facilitate and speed up LADM development by describing the standard with INTERLIS. The core work was completed in 2014 and the full model is freely available from the SLM web site (LADM country profiles for the Netherlands and Switzerland were described). By this integration, the INTERLIS tool chain can be applied to handle and implement LADM compatible country profiles in a computer-assisted manner and therefore will improve implementation efficiency and reduce the cost.

Following this initial LADM-INTERLIS integration, the LADMbased country profiles of Greece (Kalogianni et al., 2016) and Colombia (Jenni et al., 2017) were described in INTERLIS 
language. In figure 2, the LADM models described in INTERLIS are illustrated as a stack.

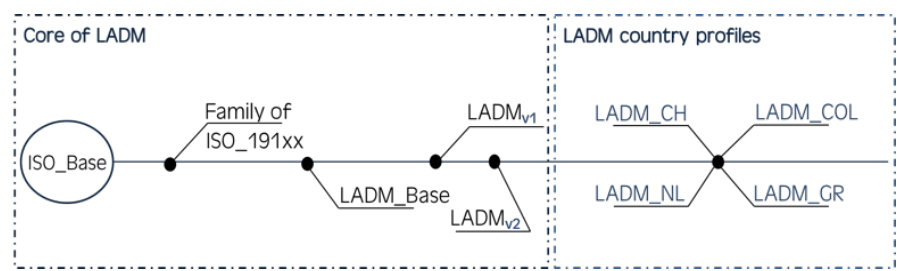

Figure 2. Stack of LADM-INTERLIS models

\subsection{Semantically enriched code lists}

Kalogianni (2016) introduced versioning and hierarchical structure in code lists described in INTERLIS language. As also mentioned by Zulkifi et al. (2014), each code list is implemented in the database with one single table. The table name has the extension "Type" after the code list name of the conceptual model. It consists of an ID (cID) for each code list and description attributes. The advantage of this type of code list is that its value can be updated and they can also be versioned when adding the attributes "beginDateTime" and "endDateTime".

Hence, code lists were designed as structures with attributes, given hierarchical structure which makes them semantically more meaningful and also extensible (Kalogianni E., 2016). The hierarchy is then added as reference to a parent code (parent_cID), which should become optionally "NULL" for root code(s). An example from the Greek implementation of code list LA_LevelContentType is given below in INTERLIS language:

STRUCTURE GR_LevelContentType $=$

cID: MANDATORY Oid; parent_cID: Oid referring to

LADM.SpatialUnit.LevelContentTypecID;

code: CharacterString;

description: CharacterString;

begin date: DateTime;

end_date: DateTime;

!! Possible code list values: (archeological, 2D parcel, 3D_parcel, mine, SRPO, planning_zone, marine2D_parcel);

END GR_LevelContentType;

The generated SQL code of the corresponding database table:

CREATE TABLE GR LevelContentType (cID integer NOT NULL PRIMARY KEY, parent_cID integer FOREIGN KEY REFERENCES,

code character varying $(50)$, gr_levelcontenttype(cID),

description character varying (255),

beginDateTime datetime,

endDateTime datetime);

Every code list has, in theory, same structure with the one presented above and therefore all code lists could be maintained in single table having an extra attribute to indicate the actual code list to which this code list value belongs.

\subsection{Formal specification of constraints}

The approach that was developed for the formal specification of constraints was that those that refer to one single object can be subdivided into requirements that must be met by each object of a class ("hard" constraints) and regulations, which in rare cases can be violated ("soft" constraints). Therefore, as presented by Kalogianni et al. (2016), hard constraints should always be true, otherwise the transaction should be cancelled and an error should appear on the user.

A sample INTERLIS code fragment from modelling constraints in INTERLIS is displayed below:

\section{CLASS GR 3DParcel EXTENDS GR Parcel = area: LIST $\{0\}$ OF GR_AreaValue; volume: LIST $\{1 . . *\}$ OF GR VolumeValue; geometry: GM_MultiSurface3D; totalSurfaceArea: Real; dimension: MANDATORY (3D); structure: MANDATORY (topological, polygon, unstructured, point);}

END GR_3DParcel;

\subsection{D data type}

LADM is based on ISO 19107, which specifies conceptual schemas for describing the spatial characteristics of geographic features and a set of spatial operations consistent with these schemas. (ISO, 2003) This model was initially translated in INTERLIS by SLM including the basic concepts, however was limited with respect to full 3D support. The second version was released in 2016 and supports both 2D and 3D geometries and 3D structures. More specifically, the 3D basic types supported by INTERLIS are GM Point3D, GM Curve3D and GM_Surface3D, while the 3D structures are GM_MultiCurve3D and GM MultiSurface3D. The authors suggested in their previous work Kalogianni et al. (2016) a definition of GM_Solid as the basic $3 \mathrm{D}$ primitive. The structure that was proposed was the following:

STRUCTURE GM Solid EXTENDS GM Object = geometry: LIST $\left\{1 .{ }^{*}\right\}$ OF GM_Surface $\overline{3}$ DListValue; END GM Solid;

In the new version of ISO 19107 described in INTERLIS, the structure GM_MultiSurface3D is defined similarly with the proposal of the authors:

GM_Curve3D = POLYLINE WITH (STRAIGHTS,ARCS) VERTEX GM Point3D WITHOUT OVERLAPS $>0.001$;

GM_Surface3D $=$ SURFACE WITH (STRAIGHTS,ARCS) VERTEX GM_Point3D WITHOUT OVERLAPS > 0.001;

STRUCTURE GM_Surface3DListValue = value: MANDATORY GM Curve3D;

END GM_Surface3DListValue;

STRUCTURE GM_MultiSurface3D = geometry: LIST $\{1 . . *\}$ OF GM_Surface3DListValue; END GM_MultiSurface3D;

\section{CONCLUSIONS AND FUTURE WORK}

The generic LADM/ INTERLIS approach can be implemented in any LADM-based model in order to get a platform independent exchange format linked to the conceptual model. Therefore, the system can be considered as a basic technological solution for Spatial Data Infrastructures related to land administration. From this integration, it is proved that the INTERLIS concept, with the development of supplementary tools can be used as an external validating mechanism for LADM-based models, as also presented by Kalogianni et al., 2016. 
Various legal characteristics and boundary types defined in specific jurisdictions can be modelled through LADM INTERLIS synergy using code list values appropriately customized for each jurisdiction; e.g. for LA_LevelContentType or LA_MonumentationType, etc.

On the other hand, also physical elements (walls, ceilings, etc.) of 3D land parcels, can be modelled in INTERLIS. A City Model in 3D has been described in INTERLIS by Swiss Land Management, however it is not based in any international standard. As a next step, CityGML or BIM/IFC could be modelled in INTERLIS language and thus, specific of spatio semantic relationships between physical objects and legal objects could be explicitly described through INTERLIS constraints.

Regarding the UML code list modelling; at INTERLIS level they become table - like, while at database level they are versioned and hierarchical code list values (stored in their own table, and used via primary key/ foreign key type of referencing). Talking about semantics in code lists, mappings for the proposed hierarchical and versioned structure should be developed. For the top-level code lists, the concept of "THINK" (i.e. top-level ontology-parent of all the code lists) could be further examined.

Another topic for future research is to examine whether by introducing adequate XML tools for INTERLIS, this will make INTERLIS/XML-files available for a wider range of applications, such as CityGML and LandXML.

Last but not least, with the ongoing LADM/INTERLIS project in Colombia, INTERLIS starts to break down the Swiss borders. This should result to the update and development of the necessary mappings between the existing INTERLIS tools in order to recognize and check the proposed structures, referring to constraints and code lists.

\section{ACKNOWLEDGEMENTS}

The authors would like to thank Michael Germann, for providing initial INTERLIS models (".ili" files) and his advice and feedback.

\section{REFERENCES}

Atazadeh, B., Kalantari, M., Rajabifard, A., 2016. Comparing Three Types of BIM-based Models for Managing 3D Ownership Interests in Multi-Level Buildings. In: $5^{\text {th }}$ International FIG 3D Cadastre Workshop, Athens, Greece.

Germann, M., Lemmen, C.H.J., Kaufmann, J., van Oosterom, P.J.M., Zeeuw, K., 2014. The LADM Based on INTERLIS. FIG Working Week 2015, Sofia, Bulgaria.

Groger, G., Kolbe, T.H., Nagel, C., Hafele, K.H., 2012. OGC City Geography Markup Language (CityGML) Encoding Standard. Open Geospatial Consortium: Wayland, MA, USA.

ISO, 2003. ISO 19107:2003 Preview Geographic information -Spatial schema. International Organization for Standardization (ISO), Geneva, Switzerland.

Janečka K., Karki S., 2016. 3D Data Management - Overview Report. In: $5^{\text {th }}$ International FIG 3D Cadastre Workshop, Athens, Greece.

Jenni, L., Germann, M. Eisenhut, C., Guarin, A., Bajo, M.V., 2017. LADM Implementation in Colombia - Process,
Methodology and Tools developed and applied. FIG Working Week 2017, Helsinki, Finland.

Kalogianni, E., Dimopoulou, E., van Oosterom, P.J.M., 2015. A 3D LADM prototype implementation in INTERLIS. In: 3D GeoInfo Conference 2015, Kuala Lumpur, Malaysia.

Kalogianni, E., 2016. Linking the legal with the physical reality of 3D objects in the context of Land Administration Domain Models. Master Thesis, Delft University of Technology, Delft, The Netherlands.

Kalogianni, E., Dimopoulou, E., Quak, W., van Oosterom, P.J.M., 2016. Formalizing Implementable Constraints in the INTERLIS Language for Modelling Legal 3D RRR Spaces and 3D Physical Objects. $5^{\text {th }}$ International FIG 3D Cadastre Workshop. FIG, Athens, Greece.

KOGIS, 2006. INTERLIS 2.3 Reference Manual. Bern, Switzerland.

Lemmen, C.H.J., van Oosterom, P.J.M, Ploeger, H., Paasch, M.J., 2014. Legal Refinement of the LADM Standard: More classes or extended code lists with better defined types of Rights, Restrictions and Responsibilities? In: PLPR 2014 Conference: Book of Abstracts.

Lemmen, C.H.J., van Oosterom, P.J.M., Bennett, R., 2015. The Land Administration Domain Model. Land Use Policy, 49, pp. $535-545$.

NBIMS, 2012. National BIM Standard - United StatesTM Version 2.

Scarponcini, P., Gruler, H. C., Stubkjær, E., Axelsson, P., Wikstrom, L., 2016. OGC® Land and Infrastructure Conceptual Model Standard (LandInfra).

Thompson, R. J., van Oosterom, P.J.M, Karki, S., Cowie, B., 2015. A Taxonomy of Spatial Units in a Mixed 2D and 3D Cadastral Database. In: FIG Working Week 2015, Sofia, Bulgaria, 2015.

Zulkifli, N, A., Rahman, A., Jamil, H., Hua, T.C., Choon T. L., Seng. L.K., Lim, C.K., van Oosterom, P.J.M, 2014. Towards Malaysian LADM Country Profile for 2D and 3D Cadastral Registration System. In: Proceedings of the 25th FIG Congress, Kuala Lumpur, Malaysia, pp. 19. 